\title{
Responsabilidade de Intermediários dentro e fora da Infraestrutura da Internet
}

\author{
Juliao Braga ${ }^{1}$, Jeferson Campos Nobre ${ }^{2}$ \\ ${ }^{1}$ Universidade Presbiteriana Mackenzie (UPM) \\ Sao Paulo - SP - Brazil \\ ${ }^{2}$ Instituto de Informática - Universidade Federal do Rio Grande do Sul (UFRGS) \\ Caixa Postal 15.064 - 91.501-970 - Porto Alegre - RS - Brazil \\ juliao@braga.net.br, jcnobredinf.ufrgs.br
}

\begin{abstract}
This article aims to analyze the intermediaries that gravitate around the Internet, identifying their location, functions and types of interference where possible damage can be caused by transmitting misinformation for lack of understanding. It locates the problems and proposes solutions through the proposal of development of a code of ethics by the multiple interested parties so that the use of cryptography can be justified, freely by whoever is interested and, especially in the relations between the origin and destination of the Internet - standardized by the Internet Engineering Task Force (IETF) - avoiding the ossification of transport protocols.
\end{abstract}

Keyworks: encryption, end-to-end encryption, traffic, transit, peering, transport

Resumo. Este artigo tem como objetivo analisar os intermediários que gravitam em torno da Internet, identificando sua localização, funções e tipos de interferências onde eventuais danos possam ser causados transmitindo informações equivocadas por falta de compreensão. Localiza os problemas e propõe soluções através da proposta de desenvolvimento de um código de ética pelas múltiplas partes interessadas para que se possa justificar o uso da criptografia, livremente por quem interessar e, em especial nas relações entre origem e destino da Internet - padronizada pelo Internet Engineering Task Force (IETF) -, evitando a ossificação de protocolos de transporte.

Palavras-chave: internet, criptografia, criptografia fim-a-fim, tráfego, transito, peering, transporte

\section{Introdução}

Internet é uma rede de redes ${ }^{1}$. Cada uma destas redes possui a característica de ser autônoma e de poder estabelecer suas próprias políticas, principalmente, as políticas de roteamento. Por esta razão ela recebe a denominação de domínio de roteamento ou, mais comumente Sistemas Autônomos (AS) (HAWKINSON, March 1996). A Figura 1 exibe uma representação parcial dos ASs conectados. Eventualmente, para representar o domínio de um AS será usado, também, um retângulo, como indicado na Figura 2.

Algumas vezes por falta de entendimento técnico mínimo, tanto por parte de pessoas como de instituições, a Internet sofre algumas pressões que podem representar \footnotetext{
$0 />$

${ }^{1} 70.123$ redes autônomos (ASs) segundo o CIDR REPORT for 5 Dec 20: <https://www.cidr-report.org/2.
} 


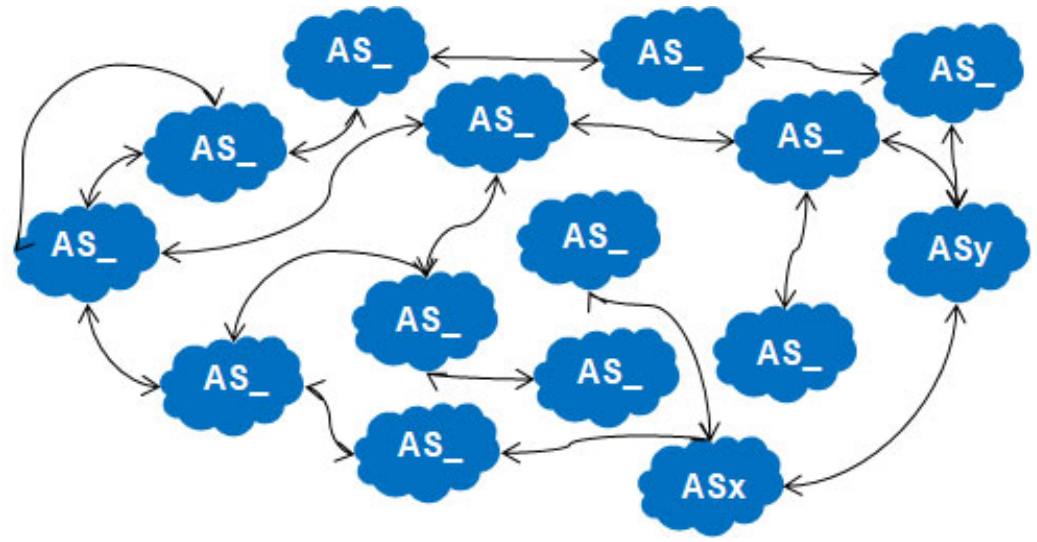

Figura 1. A Internet é formada por Sistemas Autônomos. Para $\Theta \subset \mathbb{N}$ então $\_\in$ e $, \forall i \in \Theta \quad 0 \leq i<4,294,967,296 \simeq$ $2^{32}$. Também, $x, y \in \Theta$ e $x \neq y$. Fonte: (BRAGA, 2019).

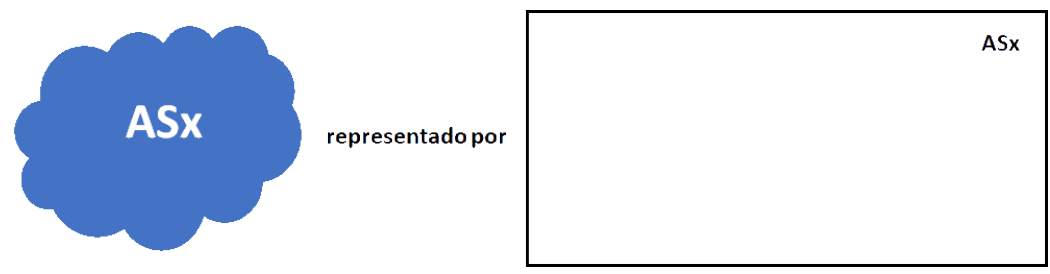

Figura 2. Pode-se representar um AS, na forma retangular, onde dentro do retângulo está toda a rede do ASx

uma ameaça ao seu funcionamento. Em geral, as partes interessadas mais diretamente envolvidas, se esforçam para diminuir este desgaste. O presente artigo segue na direção de propor o debate sobre dois pontos:

- a proibição do uso da criptografia na Internet;

- a inexistência de regras a serem respeitadas e observadas pelos provedores de mensagens instantâneas e de dados nos moldes de uma proposta para desenvolvedores de algoritmos de Inteligência Artificial (ARBIX, 2020).

O presente artigo aborda estas duas questões, discute suas aplicabilidades, analisa os conceitos equivocados por desconhecimento técnico e propões soluções. Neste contexto, procura-se evitar o debate jurídico em torno das questões relacionadas aos intermediários. Tais questões já estão bem encaminhadas e na literatura podem ser encontrados diversos exemplos (SANTOS, 2020).

Eventualmente será feito referência a três personagens fictícios: Alice, Bob e Malvino. Alice e Bob, remotamente equidistantes, sempre estarão tentando se comunicar de forma segura e Malvino é um invasor mal-intencionado, sempre pronto a adulterar as mensagens entre Alice e Bob (WIKIPEDIA, 2020). Embora as referências técnicas usem o termo datagrama para a unidade completa de dados que trafega pela Internet (MARINE; REYNOLDS; MALKIN, March 1994) este texto usará o o termo pacote para referenciar unidades de dados (completas ou não) que trafegam pela Internet, por razões puramente didáticas.

O artigo está organizado da seguinte forma. Na Seção 2 - Fundamentos, são dis- 
cutidos os conceitos importantes para o debate, incluindo suas respectivas características e definições. Na Seção 3 - A Internet e os intermediários, são identificado os intermediários, a forma como eles afetam o debate e os eventuais perigos que eles podem provocar, reduzindo a flexibilidade dos projetos de protocolos e assim afetando drasticamente o comportamento essencial da Internet. Com tais esclarecimentos pretende-se deixar claro os benefícios reais da criptografia no tráfego de trânsito e na construção de uma Internet sempre melhor. A Seção 4.1 - Os paradoxos que rondam as sociedades abertas, debate o Paradoxo da Tolerância com abordagem sobre importantes filósofos do século passado. A Seção 4 - Uma proposta para a solução dos intermediários, propõe-se uma solução, não jurídica, para ser debatida pelas múltiplas pares interessadas na importância da grande rede para a humanidade. Finalmente, na Seção 5 estabelecem-se as Conclusões para que se estabeleça o roteiro a ser considerado no debate e recomenda punir o mensageiro, se ele não respeitar as regras estabelecidas pela comunidade.

\section{Fundamentos}

Dois conceitos são importantes para o entendimento dos objetivos a serem apresentados neste artigo. Eles envolvem noções sobre tráfego e criptografia, as quais são discutidas a seguir.

\subsection{Tráfego na Internet}

Tráfego é tudo o que se movimenta nos meios físicos de transmissão de dados, através dos protocolos da Internet. Seja lá o que os bits representem é denominado de tráfego (BRAGA, 2010). Para atender ao que será dito neste artigo identificam-se três tipos de tráfego:

- Trânsito: é o tráfego trocado entre os ASs. Isto é, o tráfego de pacotes que seguem na direção da Internet. As medições de tráfego na Internet recaem sobre o trânsito, pois é o tráfego público.

- Transporte: é o tráfego que não segue na direção da Internet e geralmente é o tráfego trocado entre dois pontos de conexão quaisquer.

- Peering: é o tráfego trocado em um ambiente, sem custo. De um modo geral é o tráfego trocado entre um AS e um Ponto de Troca de Tráfego ou IX.

Para ilustrar estas três tipos de tráfego vamos supor que o $\mathbf{A S z}$ seja um provedor de conteúdo, por exemplo, filmes. E que o ASx seja um provedor de acesso à Internet com, digamos, aproximadamente 30.000 usuários interessados no conteúdo do ASz. Inicialmente, no momento $\mathbf{t}_{0}$, o acesso ao conteúdo do $\mathbf{A S z}$ é feito pela Internet, como mostra a Figura 7.(a). Esta alternativa de acesso, pelo tráfego de trânsito é a mais cara e paga pelo ASx que, na realidade cobra de seus usuários. Os usuários de $\mathbf{A S x}$, por outro lado, pagam ao $\mathbf{A S z}$ pelo acesso aos filmes.

O ASx, preocupado com os custos de trânsito para atender a demanda de acesso ao ASz solicita uma reunião para discutirem esta questão e chegam a conclusão que podem resolver este problema de duas maneiras: via tráfego de transporte dedicado ou $\mathbf{A S z}$ instalaria uma cópia de seus servidores de conteúdo, dentro das instalações do ASx. Resolvem começar com o transporte (Figura 7.(b)) e assim que o ASz estiver preparado, encaminharão a segunda solução (Figura 7.(c)). A primeira solução reduziu o custo operacional e a segunda solução reduziram os custos do ASx para zero! 


\section{Internet}

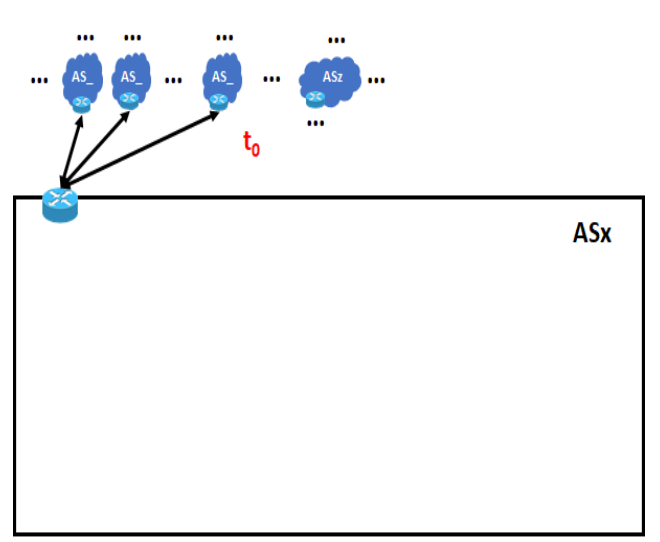

- ASz: Provedor de dados. Ex.: filmes

- $\mathrm{t}_{0}$ : Acesso ao ASz no primeiro momento, via trânsito

Figura 3. A troca de tráfego com o ASz é feita via trânsito, isto é, via Internet! É a solução mais cara.

\section{Internet}

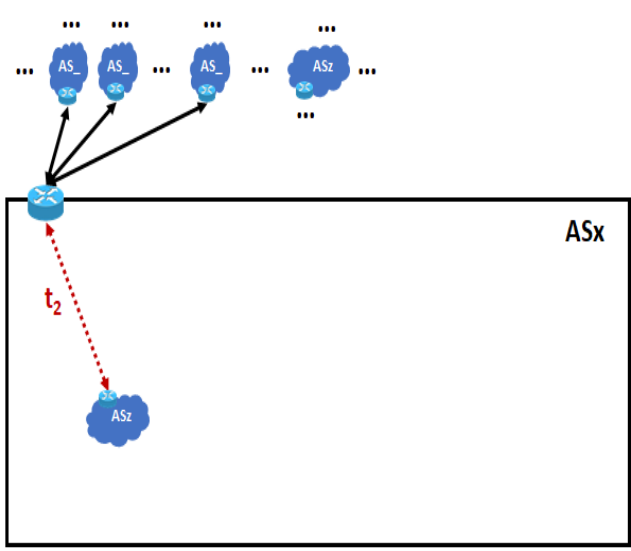

- $t_{2}:$ Acesso ao ASz em um terceiro momento, via LAN em peering

Figura 5. A troca de tráfego com o $\mathbf{A S z}$ é feita via rede local e peering. Custo zero: sem Internet e sem transporte!
I n t e r n e t

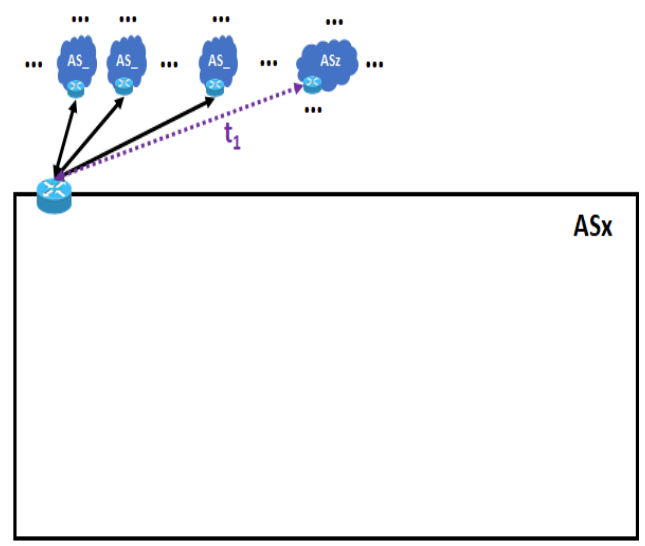

$\mathrm{t}_{1}$ : Acesso ao ASz em um segundo momento, via transporte

Figura 4. A troca de tráfego com o $\mathbf{A S z}$ é feita via transporte. O tráfego não compete com o tráfego da Internet e o custo é significativamente mais barato do que o da Figura 7.(a)!

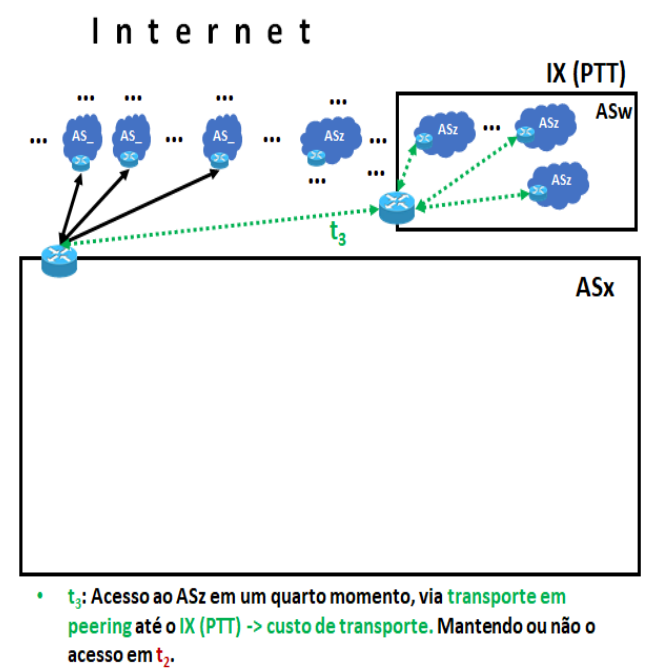

Figura 6. A troca de tráfego com o $\mathbf{A S z}$ é via transporte até um ponto de troca de tráfego (IX) (em peering), que é um AS (ASw) e hospeda outros provedores de serviços especializados (Facebook, Google, Netflix, etc.). Sem Internet!. $O$ custo é semelhante ao da Figura 7.(b) com a vantagem de acesso a muitos provedores de conteúdo.

Figura 7. Os diversos tipos de tráfego e o interesse dos intermediários. Os melhores locais são aqueles onde não há tráfego da Internet. 
Embora a segunda solução seja a melhor alternativa, a disponibilidade de intermediários ofertando conteúdos para os usuários da Internet tem aumentado e algumas vezes para implementar soluções locais de espelhamento exigem investimentos iniciais elevados e a manutenção do conteúdo pode exigir tráfego de trânsito. No caso específico do Brasil, são muitos os ISPs que demandam os provedores de conteúdo e um grande número não possui o número de usuários mínimos que justifiquem instalação local. Considerando a demanda e tais ponderações fizeram com que os responsáveis pela governança da Internet em âmbito nacional propusessem a criação de um ambiente apropriado para troca de tráfego de peering a partir de conexões via tráfego de transporte. Tais ambientes foram denominados Pontos de Troca de Tráfego (IX ${ }^{2}$ ou PTT). Esta ideia exibida na Figura 7.(d) foi um sucesso e tem sido cada vez mais procurada. No Brasil os IX são em número de 33 e andam experimentando demanda de tráfego cada vez maiores ${ }^{3}$.

Fisicamente, um AS não está dentro do ambiente do IX, mas sim conectado ao IX, usualmente via fibra ótica e através de um provedor de serviços de conexão ao IX, chamado Ponto de Interconexão ${ }^{4}$ (PIX). Adicionalmente, um membro de um IX pode-se aproveitar de conexões à Internet ofertado por outros membros do IX.

\subsection{Criptografia}

Kurose e Ross (2017) propões quatro propriedades desejáveis para uma comunicação segura:

A Confidencialidade: somente a origem e o destino devem estar habilitados ao acesso à mensagem.

B Integridade da mensagem: A origem e o destino esperam que o conteúdo da mensagem não será alterado, por má fé ou por acidente.

C Autenticação de ponto final: Cada um dos participantes da comunicação, origem e destino devem estar habilitadas a confirmar a cada um, que a outra parte é realmente quem afirma ser.

D Segurança operacional: Para garantir esta propriedade, intermediários são usados extensivamente, como por exemplo firewalls e Intrusion Detection Systems (IDS).

As três primeiras propriedades acima, (A, B e C), são diretamente dependentes de criptografia. A última propriedade (D) é garantida por intermediários e será relembrada na seção relacionada. Considerando o presente artigo, Alice e Bob acreditam que estas quatro propriedades estarão presentes durante as atividades de comunicação entre eles e, ainda, que as propriedades os protegerão de Malvino.

Diversos textos podem ser indicados para o estudo de fundamentos de criptografia ((KONHEIM, 1981), Stallings (2015), (SINGH, 2005), Kurose e Ross (2017)). Para um entendimento rápido e didático recomenda-se a Cartilha de Segurança para a Internet ${ }^{5}$, do CERT.br.

\footnotetext{
${ }^{2}$ Internet eXchange point

${ }^{3}<$ https://ix.br/trafego/agregado/vix $>$

${ }^{4}<$ https://ix.br/trafego/pix/>

${ }^{5}$ https://cartilha.cert.br/criptografia/
} 


\subsubsection{Criptografia forte, criptografia fraca}

Há um aparente equívoco quando se associa criptografia forte a criptografia fim-a-fim (POLK, 2020). Este equívoco pode levar a um entendimento incompleto sobre o valor da criptografia e eventuais interpretações de sua efetiva garantia de preservar as quatro propriedades de Kurose (item 2.2). Da mesma forma, quando se considera que que o tamanho da chave usada pelo algoritmo de criptografia é quem define uma criptografia forte ou fraca.

Bruce Schneier faz as seguintes observações sobre criptografia forte / fraca e respectivos algoritmos, após argumentar que criptografia forte é muito poderosa quando feita corretamente (SCHNEIER, 1998):

Um sistema criptográfico só pode ser tão forte quanto os algoritmos de criptografia, algoritmos de assinatura digital, funções hash unidirecionais e códigos de autenticação de mensagens dos quais depende. Quebre qualquer um deles, e você quebrará o sistema. E assim como é possível construir uma estrutura fraca usando materiais fortes, é possível construir um sistema criptográfico fraco usando algoritmos e protocolos fortes.

Sob o ponto de vista prático, qualquer criptografia é forte, principalmente nos dias atuais em que os processos de obtenção da chave única (na criptografia simétrica) e de obtenção das chaves públicas e privadas, facilitam e garantem que o tamanho da chave seja escolhido pelo interessado, adaptando-a à aplicação.

\subsubsection{Criptografia Simétrica}

Criptografia simétrica é a técnica de criptografar usando uma única chave. A chave usada para criptografar é a mesma usada para descriptografar. Não é uma técnica recomendada para um processo de criptografia envolvendo mais de um participante, pois compartilhamento de chave não é um método seguro, principalmente se a origem e destino estão remotamente localizado. Mas é apropriada para criptografar conteúdos locais considerando que a chave única não sairá do ambiente local, onde o algoritmos de criptografia é usado.

Em geral, o processo de criptografar usando uma chave única é sensivelmente mais demorado. Existem diversos algoritmos que usam a chave simétrica (KUROSE; ROSS, 2017): (a) Data Encryption Standard (DES), usa chave de 56 bits; (b) Advanced Encrypton Standard (AES), pode usar chaves com 128, 192 e 256 bits de tamanho.

\subsubsection{Criptografia Assimétrica}

Criptografia assimétrica é a técnica de criptografar usando duas chaves: pública e privada. Se Alice tem de enviar uma mensagem para Bob, Alice precisa de descobrir qual a chave pública de Bob para usá-la no processo de criptografar. Ao fazer isto, o texto criptografado por Alice somente será decriptografado se for usada a chave privativa de Bob.

Esta técnica de criptografia é eficaz para os casos em que a origem e destino estão remotamente separados e é usada, também, no chamado processo de criptografia 
fim-a-fim. Há vários algoritmos para obter uma chave pública e a respectiva chave privada associada. Dois se destacam: (a) Rivest, Shamir and Adleman (RSA) (RIVEST; SHAMIR; ADLEMAN, 1978); (b) Diffie-Hellman Key Exchange (Diffie-Hellman) (DIFFIE; HELLMAN, 1976).

Na sequência são destacadas três aplicações da criptografia assimétrica, consideradas bastante úteis e usuais.

PGP Pretty Good Privacy (PGP) foi escrito por Philip Zimmermann ${ }^{6}$ em 1997 e padronizado pelo IETF em 2007 como OpenPGP ${ }^{7}$ (CALLAS et al., November 2007). A Figura 8 exibe as etapas que preenchem o processo de criptografia de uma mensagem criptografada, enviada por Alice para Bob usando o PGP e o envolvimento direto das chaves pública e privada, do Bob.

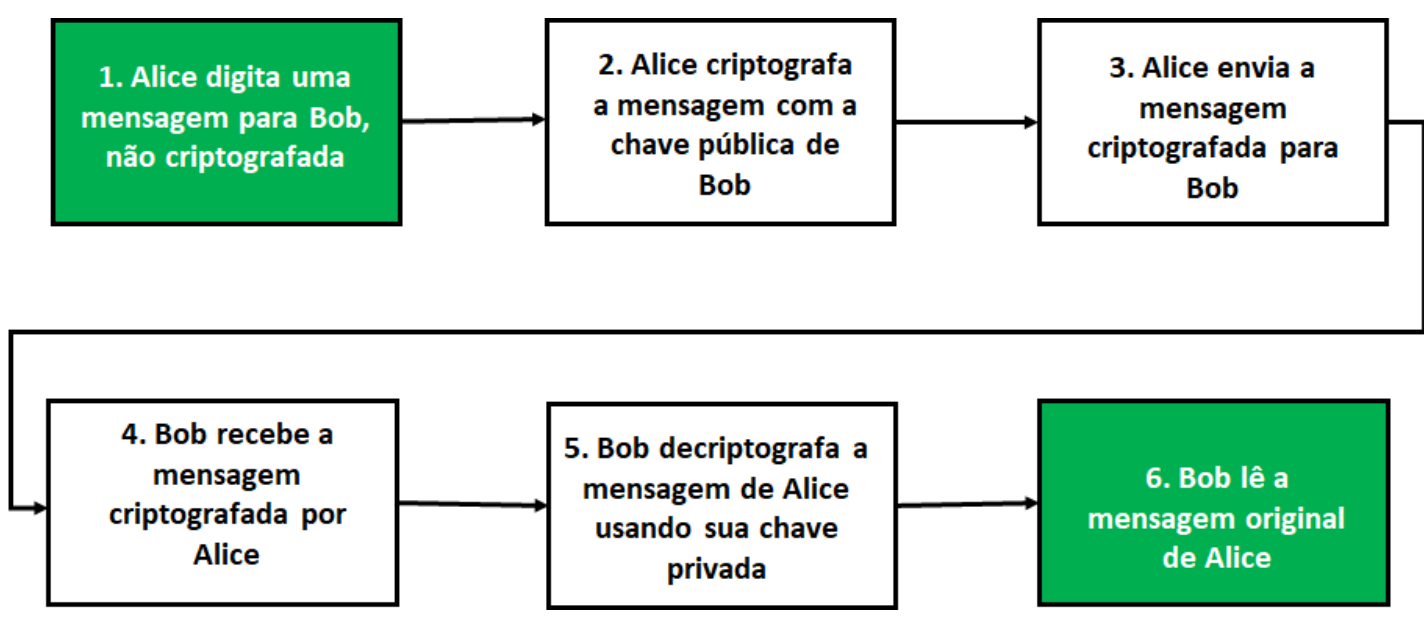

Figura 8. PGP: uma aplicação da criptografia assimétrica. Alice criptografa uma mensagem para Bob, usando a chave pública de Bob. Só Bob pode decriptografar com sua chave privada. O tráfego de trânsito (3-> 4) está seguro.

As etapas são executadas automaticamente pelo software cliente de e-mail usado por Alice. Ela descobre a chave pública de Bob, armazenada em algum servidor especializado ou localmente, quando esta não for a primeira mensagem enviada a Bob. No destino, o software de Bob confirma se a mensagem veio de Alice e se foi criptografada usando a chave pública de Bob. Se houve alguma interferência no trânsito, a mensagem não consegue ser decriptografada e Alice repetirá o procedimento, se for o caso.

Transport Layer Security Transport Layer Security (TLS) é uma técnica de criptografia fim-a-fim e sucessor da famosa técnica Secure Sockets Layer (SSL) em sua versão 3 (RESCORLA, August 2018). Padronizada pelo IETF (DIERKS; RESCORLA, April 2006), o TLS, em sua versão 1.3 reduziu significativamente o tempo para estabelecer as etapas de preparo, que antecedem, por exemplo, o pedido de transferência de uma página de Web através do protocolo HTTPS $^{8}$ pelo cliente. Quando o TLS é utilizado, um intermediário

\footnotetext{
${ }^{6}<$ https://philzimmermann.com/EN/background/index.html>

${ }^{7}<$ https://www.openpgp.org>

${ }^{8} \mathrm{O}$ protocolo HTTPS é uma das aplicações do TLS, que é usado em outros protocolos, como por exemplo no processo de autenticação do EDUROAM (BRAGA et al., 2020)
} 
somente enxerga os pontos de conexão (origem e destino), o tipo de criptografia utilizado, a frequência e a quantidade aproximada de dados enviado, mas não consegue ler ou modificar os dados reais ${ }^{9}$.

O processo de preparação e a troca de chaves na sequência é denominado handshake, pode ser visto na Figura 9.

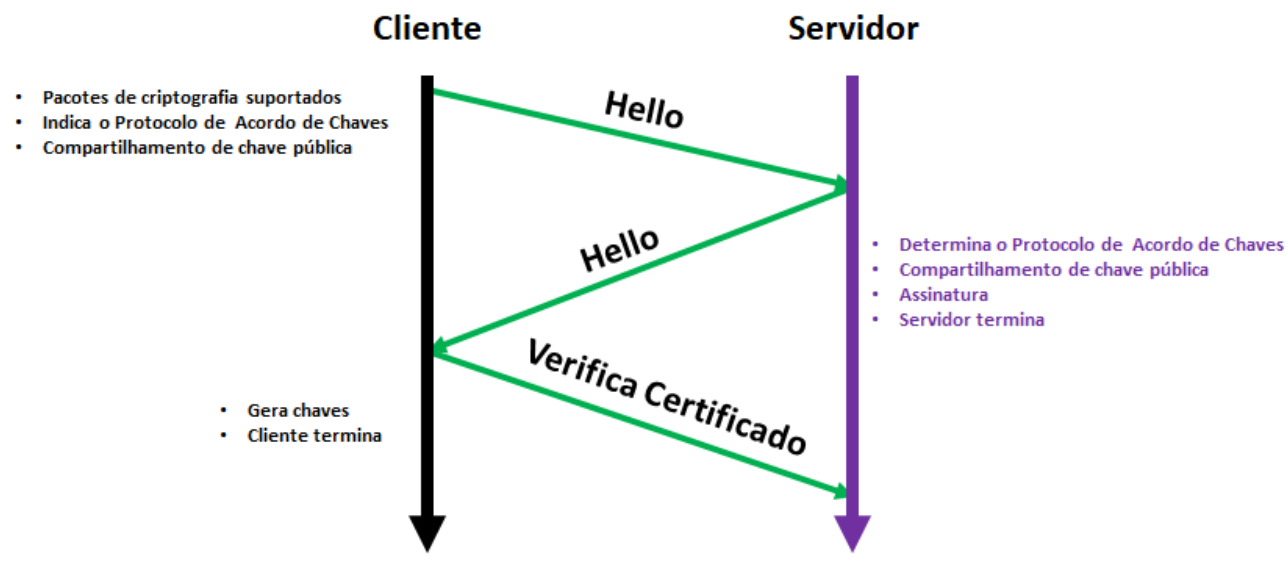

Figura 9. O aperto de mão (handshake) do TLS 1.3. O Cliente indica duas técnicas para acordo (de troca) de chaves: RSA ou Diffie-Hellman. Adaptado de Rescorla (August 2018).

O detalhamentos das etapas do handshake mostrado na figura acima foge do escopo do presente artigo mas, seu comportamento pode ser compreendido no detalhamento da RFC8446 Rescorla (August 2018). Complementarmente, há uma interpretação daquela RFC, no The Cloudflare Blog ${ }^{10}$. Vale notar que o TLS 1.3, durante sua etapa de handshake, indica dois algoritmos para troca de chaves, a ser escolhido pelo Servidor, através dos respectivos certificados: RSA ou Diffie-Hellman ${ }^{11}$.

Internet Protocol Security - (IPSec) O PGP criptografa uma mensagem, como se pode observar no exemplo acima. O TLS mostra a criptografia de um soquete em uma aplicação cliente-servidor. O IPSec criptografa TODO o tráfego entre dois dispositivos. Uma aplicação, muito comum, do IPSec é quando do estabelecimento de uma Virtual Private Network, principalmente, entre dispositivos que trocam tráfego de trânsito. É comum o uso de IPSec entre máquinas que trocam trânsito (IPSec máquina a máquina). A arquitetura da segurança existente no IPSec esta descrita na RFC4301 (KENT; SEO, December 2005) e os detalhes de todo o protocolo estão caracterizados na RFC6071 (FRANKEL; KRISHNAN, February 2011).

Dois protocolos se sobressaem no ambiente IPSec: Authentication Header (AH) e o Encapsulation Security Payload (ESP). Quando a origem envia um pacote seguro para o destino, ela usa o AH ou o ESP. O protocolo AH garante a autenticação da fonte e a integridade dos dados mas disponibiliza a confidencialidade. O protocolo ESP

\footnotetext{
${ }^{9}<$ https://hpbn.co/transport-layer-security-tls/>

${ }^{10}<$ https://blog.cloudflare.com/rfc-8446-aka-tls-1-3/>

${ }^{11}<$ https://en.wikipedia.org/wiki/Diffie-Hellman_key_exchange>
} 
disponibiliza a autenticação da fonte, a integridade dos dados e a confidencialidade. Comparando um com outro vê-se que o ESP é mais usado do que o AH (KUROSE; ROSS, 2017).

\section{A Internet e os intermediários}

São muitos os intermediários presentes na Internet. A cada dia, hora ou segundo aparecem e desaparecem intermediários. Alguns são AS (Sistemas Autônomos) e possuem caráter permanente, outros não são AS, mas fazem parte da rede de um Sistema Autônomo. Para efeito didático dividiu-se os intermediários em dois tipos: intermediários externos e intermediários internos. Eles estão apresentados na Figura 10.

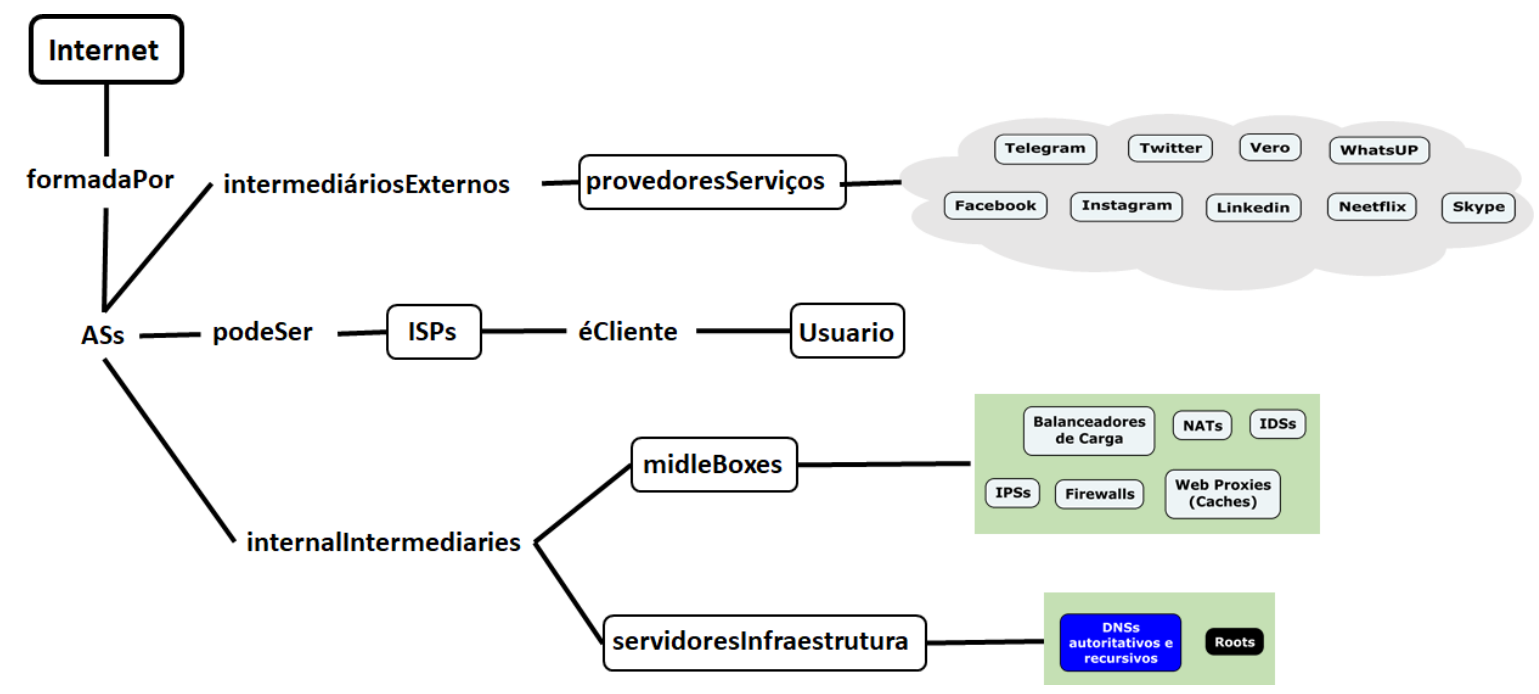

Figura 10. Onde estão e quem são os intermediários no contexto da Internet

Os intermediários externos são, basicamente, os fornecedores de serviços que em sua grande maioria são ASs. Embora sua oferta de serviços é feita via tráfego de trânsito, eles procuram estar presente nos IXs, o que torna o custo de acesso a seus serviços, consideravelmente mais baixo. Esta independência faz com que sua representação esteja em nuvem (ilustrada na Figura 10). Os intermediários internos estão presentes internamente na rede de um AS. Eles são apresentados em dois tipos: as caixas intermediárias ou middle boxes e os servidores de infraestruturas.

Os servidores de infraestruturas estão representados para mostrar que são intermediários que não representam perigo para a Internet. Pelo contrário são intermediários fundamentais para o funcionamento da Infraestrutura da Internet de um país. Dois deles estão representados: os servidores de DNS (autoritativos ou recursivos) e os servidores roots. O primeiro é um dos mais importantes intermediários da Internet e está bem desenvolvido sob o ponto de vista da segurança de suas funções. Uma técnica de criptografia assimétrica garante a integridade das informações que ele passa aos clientes de DNS. Os servidores de root representam a peça de suporte aos servidores de DNS e com um grande número de espelhamento espalhados pelo mundo garante a segurança operacional da resolução de nomes tornando inviável o ataque de terceiros interessados em ofender o bom funcionamento da Internet. Adicionalmente, a presença de servidores root em maior ou menor número em outros países não representam agressão à soberania distribuída da Internet. 
As caixas intermediárias podem representar, com frequência, um perigo para a Internet e serão impedidos através do uso da criptografia, em sua maioria, a criptografia fim-a-fim.

\subsection{Caixas intermediárias}

A definição de caixas intermediárias ou middleboxes é:

Uma caixa intermediária é definido como qualquer dispositivo intermediário que executa funções diferentes das funções normais e padrões de um roteador IP no caminho do pacote entre um dispositivo de origem e um dispositivo de destino (SRISURESH et al., August 2002; CARPENTER; BRIM, February 2002).

As caixas intermediárias interferem no pacote TCP/IP durante sua passagem pelo ambiente de um AS. Algumas destas caixas são bem conhecidas e os padrões do Internet Engineering Task Force (IETF) regulam seu comportamento através de RFCs (IETF, 2020; RFC Editor, 2020). Outras, não são reguladas e são ofertadas pelo mercado de desenvolvedores, sem que se saiba exatamente os detalhes de seus algoritmos mas com promessas de eficiência e controle adicional, de interesse dos administradores de ASs.

Quando uma caixa intermediária faz uma mudança no pacote do TCP/IP, contrariando os padrões estabelecidos pelo IETF o projeto do protocolo envolvido perde sua flexibilidade e causa uma disfunção na Internet conhecida como ossificação do protocolo, o qual se discute na subseção seguinte.

Em sua maioria os protocolos afetados estão na camada de transporte. Este protocolos são responsáveis pela confiabilidade e integridade além de especificarem qual o destino (aplicação) o conjunto de pacotes deverá ser dirigido.

\subsubsection{A ossificação dos protocolos de transporte, as middleboxes e a criptografia}

Ossificação de protocolo é uma redução progressiva na flexibilidade do projeto do protocolo de rede causada pela presença de middleboxes na rede, que não podem ser facilmente removidos ou atualizados para permitir alterações de protocolo.

Mudanças no TCP também sofrem ossificação: algumas caixas entre um cliente e o servidor remoto irão detectar novas opções de TCP desconhecidas e bloquear tais conexões, já que eles não sabem quais são as opções. Se puderem detectar detalhes do protocolo, os sistemas aprenderão como os protocolos normalmente se comportam e, com o tempo, torna-se impossível alterá-los. A única maneira verdadeiramente eficaz de "combater"a ossificação é criptografar o máximo possível da comunicação para evitar que as caixas intermediárias vejam muito do protocolo passando ${ }^{12}$.

Uma metodologia relatada por Edeline (November 2020) procurando por caixas intermediárias em cerca de 52,8 milhões de caminhos, descobriu-se que 20,5 milhões $(38,9 \%)$ de caminhos estão cruzando pelo menos uma caixa intermediária. A metodologia foi definida por Detal et al. (2013) e os resultados foram os seguintes, onde entre os caminhos percorridos:

\footnotetext{
${ }^{12}<$ https://http3-explained.haxx.se/en/why-quic/why-ossification>
} 
- 32,4\% incluem uma caixa intermediária benigna.

- $6,5 \%$ são potencialmente prejudicados.

- $0,1 \%$ envolvem uma caixa intermediária que bloqueia o tráfego, que na ausência de um mecanismo de fallback, resulta em uma falha de conectividade.

- $0,8 \%$ estão interrompidos por apresentarem deficiências múltiplas, ou seja, possuem dois ou mais recursos desativados interrompendo o tráfego e a negociação.

- 5,6\% são afetados por caixas intermediárias de interrupção de tráfego.

\section{Uma proposta para a solução dos intermediários}

Diante dos fatos expostos, e em exemplos aplicados como, o protocolo QUIC ${ }^{13}$, no qual se usou criptografia para evitar as caixas intermediárias ${ }^{14}$ a solução parece ser, realmente, a criptografia que além de evitar a ossificação garante a presença das quatro propriedades (A, B, C, D), propostas por Kurose (seção 2.2).

A proposta é portanto, evitar qualquer impasse que possa conduzir ao crescimento das vozes e esforços para eliminar a criptografia da Internet. Tal preocupação deve ser global. Os produtores de caixas intermediárias devem se ater às especificações das propostas produzidas pelas organizações que cuidam do desenvolvimento de padrões, como o IETF. Até porque, as caixas intermediárias poderão frustrar seus objetivos com as implementações cada vez mais apropriadas e eficazes das técnicas de criptografia fim-a-fim.

Imprescindível a necessidade de esclarecer a importância de garantir a confidencialidade de mensagens trocadas entre indivíduos. Como consequência deve ficar claro que o mensageiro não deve ser punido (ISOC, 2020). Mas o mensageiro deve se juntar ao esforço da proposta do parágrafo anterior.

\subsection{Os paradoxos que rondam as sociedades abertas}

A tolerância ilimitada leva ao desaparecimento da tolerância. Este é o paradoxo ((BUNCH, 2012)) estabelecido em 1945 pelo grande filósofo contemporâneo Karl Popper (POPPER, 2020). Popper esclarece: "se estendermos a tolerância ilimitada mesmo aos intolerantes, e se não estivermos preparados para defender a sociedade tolerante do assalto da intolerância, então, os tolerantes serão destruídos e a tolerância com eles". Os intermediários, com sua poderosa presença na Internet tendem a imporem seus interesses, de forma intolerante e sem respeito sobre os que lhe dão o poder.

Em 1971, o filósofo Paul Rawls concluiu em sua obra Theory of Justice, que uma sociedade justa deve tolerar o intolerante, caso contrário, a sociedade seria então ela própria intolerante, e portanto injusta (RAWLS, 2009). Rawls também insiste, como Popper, que a sociedade tem um direito razoável de auto-preservação que supera o princípio da tolerância: "ao passo que uma seita intolerante não possui pretexto para reclamar de intolerância, a sua liberdade deve ser restringida em relação aos tolerantes somente quando estes últimos creem que a sua própria segurança e as instituiçõos que preservam a liberdade estão em perigo".

\footnotetext{
${ }^{13} \mathrm{Em} 2017$ o QUIC representou 7\% do tráfego total da Internet: <https://blog.apnic.net/2018/05/15/ how-much-of-the-internet-is-using-quic/>

${ }^{14}<$ https://blog.cloudflare.com/the-road-to-quic/>
} 


\section{Conclusões}

Arbix (2020) observa que "A Inteligência Artificial (IA) se configura como uma constelação de tecnologias, capaz de gerar outras tecnologias, novas metodologias e aplicações e, por isso, suas características são de natureza distinta da de outras inovações. Seu desenvolvimento propõe questões relacionadas à ética de seus algoritmos, o que envolve processos de decisão nem sempre pautados pela transparência, contrastando por vezes com direitos de indivíduos e valores das sociedades."No final de seu texto lembra que a Declaração de Toronto ${ }^{15}$ proposta em 2018 em defesa dos sistemas de aprendizagem de máquinas. Tal proposta pode ser, perfeitamente adaptada, às questões que envolvam intermediários.

As múltiplas partes interessadas, produtores de caixas intermediárias, prestadores de serviços intermediários e os próprios usuários da Internet e intermediários, incluindo instituições e organizações, também interessados em garantir que a Internet funcione cada vez melhor, precisam estabelecer em conjunto, propostas de códigos de conduta associados com o intermediário - regras éticas, por exemplo. Tais propostas devem possuir indicações e interpretações das leis mais gerais, como a Constituição e leis específicas, como o Marco Civil da Internet Brasileira, além das recomendações de comportamentos que o intermediário e os produtores de caixas intermediárias devem aceitar e se comprometer a adotar quando desenvolver algoritmos, técnicas ou aplicações no país que ele desejar se estabelecer. A criação de, por exemplo, fundamentos éticos a serem respeitados por aqueles que adotam a intolerância no estabelecimento de regras unilaterais é uma oportunidade de seguir a proposta de um salto na ciência, com a criação de paradigmas, recomendado pelo não menos brilhante filósofo contemporâneo, Thomas Kuhn (KUHN, 1996).

Se o intermediário não respeitar as regras assim estabelecidas, então ele deve ser punido. Observa-se que no início do ano de 2021, diversos intermediários, à revelia da comunidade e intolerantemente, puniram um outro intermediário, o Parler com argumentos de ausência de uma ética criada por um estreitíssimo ambiente de partes interessadas.

\section{Trabalhos futuros}

As atividades que podem garantir o avanço sobre as ideias aqui propostas envolvem, principalmente, recomendações às instituições que se preocupam, diretamente, com a governança da Internet e, em particular, com direitos humanos digital. Adicionalmente seria oportuno o desenvolvimento de um esboço técnico preliminar com tópicos justificados, como forma de alavancagem dos debates.

\section{Conflitos de interesses}

Os autores declaram não haver interesses conflitantes.

\section{Referências}

ARBIX, G. A Transparência no centro da construção de uma IA ética. Novos estudos CEBRAP, SciELO Brasil, v. 39, n. 2, p. 395-413, 2020.

\footnotetext{
${ }^{15}$ The Toronto Declaration: Protecting the right to equality and non-discrimination in machine learning systems (2018). Disponível em: <https://www.torontodeclaration.org/declaration-text/english>,
} 
BRAGA, J. Tráfego, trânsito, transporte e peering: uma proposta de definição. 2010. [Online; acessado em 23-dezembro-2020]. Disponível em: <https://ii.blog.br/2010/03/09/ trafego-transito-transporte-e-peering/>.

BRAGA, J. Ambiente para Aquisição de Conhecimento por Agentes em Domínios Restritos na Infraestrutura da Internet. Tese (Doutorado) - Instituto Superior Técnico \& Universidade Presbiteriana Mackenzie, 2019.

BRAGA, J. et al. Diminuindo a desigualdade no acesso à Internet entre estudantes alfabetizados até a entrada na Universidade. In: SBC. Anais do VI Workshop Pré-IETF To be published. [S.1.], 2020.

BUNCH, B. Mathematical fallacies and paradoxes. [S.1.]: Courier Corporation, 2012.

CALLAS, J. et al. OpenPGP Message Format. [S.1.], November 2007. DOI: 10.17487/RFC4880.

CARPENTER, B.; BRIM, S. Middleboxes: Taxonomy and Issues. [S.1.], February 2002. DOI: $10.17487 /$ RFC3234.

DETAL, G. et al. Revealing middlebox interference with tracebox. In: Proceedings of the 2013 conference on Internet measurement conference. [S.1.: s.n.], 2013. p. 1-8.

DIERKS, T.; RESCORLA, E. The Transport Layer Security (TLS) Protocol Version 1.1. [S.1.], April 2006. DOI: 10.17487/RFC4346.

DIFFIE, W.; HELLMAN, M. New directions in cryptography. IEEE transactions on Information Theory, IEEE, v. 22, n. 6, p. 644-654, 1976.

EDELINE, K. TCP path brokenness and transport layer evolution. November 2020. APNIC. Disponível em: <https://blog.apnic.net/2020/11/03/ tcp-path-brokenness-and-transport-layer-evolution/>.

FRANKEL, S.; KRISHNAN, S. IP Security (IPsec) and Internet Key Exchange (IKE) Document Roadmap. [S.1.], February 2011. DOI: 10.17487/RFC6071.

HAWKINSON, J. Guidelines for creation, selection, and registration of an Autonomous System (AS). [S.1.], March 1996. RFC1930. (DOI: 10.17487/RFC1930).

IETF. Internet Engineering Task Force. 2020. [Online; acessado em 23-dezembro-2020]. Disponível em: <https://ietf.org>.

ISOC. Internet Way of Networking Use Case: Intermediary Liability. 2020. Disponível em: <https://www.isoc.org.br/files/ d-Internet-Way-of-Networking-Use-Case-Intermediary-Liability.pdf>.

KENT, S.; SEO, K. Security Architecture for the Internet Protocol. [S.1.], December 2005. DOI: $10.17487 / \mathrm{RFC} 4301$.

KONHEIM, A. G. Cryptography, a primer. [S.1.]: John Wiley \& Sons, Inc., 1981.

KUHN, T. S. A Estrutura das Revoluções Científicas. 1. ed. São Paulo: Perspectiva, 1996.

KUROSE, J. F.; ROSS, K. W. Computer networking: a top-down approach. 7. ed. [S.1.]: Pearson Education Limited, 2017. 
MARINE, A.; REYNOLDS, J.; MALKIN, G. FYI on Questions and Answers Answers to Commonly asked "New Internet User"Questions. [S.1.], March 1994. DOI: 10.17487/RFC1594.

POLK, R. EU Internet Society Chapters Call on European Commission to Follow the Path of Strong Encryption. Here's Why You Should Too. 2020. Accessed 22 Dec 2020. Disponível em: <http://bit.ly/rpolk>.

POPPER, K. R. The open society and its enemies: Hegel and Marx. [S.1.]: Princeton University Press, 2020. v. 119.

RAWLS, J. A theory of justice. [S.1.]: Harvard university press, 2009.

RESCORLA, E. The Transport Layer Security (TLS) Protocol Version 1.3. [S.1.], August 2018. DOI: $10.17487 /$ RFC8446.

RFC Editor. Request for Comment Editor. 2020. [Online; acessado em 23-dezembro-2020]. Disponível em: <https://rfc-editor.org $>$.

RIVEST, R. L.; SHAMIR, A.; ADLEMAN, L. A method for obtaining digital signatures and public-key cryptosystems. Communications of the ACM, ACM New York, NY, USA, v. 21 , n. 2, p. 120-126, 1978.

SANTOS, B. M. dos. Uma avaliação do Modelo de Responsabilidade de Intermediários do Marco Civil para o desenvolvimento da Internet no Brasil. 2020. Disponível em: $<$ https://isoc.org.br/files/1_5163560127365644511.pdf>.

SCHNEIER, B. Security pitfalls in cryptography. In: THE EDI GROUP, LTD. EDI FORUM-OAK PARK-. [S.1.], 1998. v. 11, p. 65-69.

SINGH, S. O Livro dos Códigos. 5. ed. São Paulo: Editora Record, 2005.

SRISURESH, P. et al. Middlebox communication architecture and framework. [S.1.], August 2002. DOI: 10.17487/RFC3303.

STALlingS, W. Criptografia e Segurança de Redes: Princípios e Práticas. 6. ed. São Paulo, Brasil: Pearson Education do Brasil, 2015.

WIKIPEDIA. Alice e Bob - Wikipedia, a enciclopédia livre. 2020. [Online; acessado em 23-dezembro-2020]. Disponível em: <https://pt.wikipedia.org/w/index.php?title=Alice_e_ Bob\&oldid $=54652313>$. 